\title{
CYCLIC ORDER AND DISSECTION ORDER OF CERTAIN ARCS
}

\author{
S. B. JACKSON
}

\begin{abstract}
Let $\operatorname{arc} A$ in the conformal plane or on the sphere have local cyclic order three and cyclic order $t$. It can be decomposed into a finite number of subarcs of cyclic order three. Let the disection order of $A$ be the minimum number of arcs in such a decomposition. The principal result of this paper is that the cyclic order $t$ and dissection order $d$ of $A$ satisfy the relation $d+2 \leqq t \leqq 3 d$. In establishing this result it is proved that a necessary and sufficient condition, that an arc of local cyclic order three shall be of global cyclic order three, is that there exists a circle meeting it only at the endpoints.
\end{abstract}

1. Introduction. Let arc $A$ of the conformal plane or sphere have local cyclic order three, i.e., let every point have a neighborhood on $A$ meeting any circle at most three times. A circle $C$ containing a point $p \in A$ is called a general tangent circle to $A$ at $p$ if $C=\lim C\left(q_{i}, r_{i}, P_{i}\right)$ where $\left\{q_{i}\right\}$ and $\left\{r_{i}\right\}$ converge on $A$ to $p,\left\{P_{i}\right\}$ converges, and $C\left(q_{i}, r_{i}, P_{i}\right)$ denotes the unique circle through the three points. If $\left\{P_{i}\right\}$ also converges on $A$ to $p$, then $C$ is called a general osculating circle to $A$ at $p$. Since $A$ has cyclic order three at each point $p$, the general tangent circles form a unique pencil of the second kind with fundamental point $p([4]$, [9]). From the Heine-Borel Theorem it follows that $A$ has finite cyclic order. The following notation will be used throughout the paper:

$A$ is an arc of local cyclic order 3 .

$a, b$ are the endpoints of $A$.

$A^{\prime}=A \backslash\{a, b\}$, i.e. $A^{\prime}$ consists of the interior points of $A$.

$t$ is the cyclic order of $A$.

$\boldsymbol{K}$ is the pencil of circles through points $a, b$.

The following result is known [6, Theorem 1].

TheOREM A. The general osculating circles to arc A have the nesting property.

Received by the editors May 19, 1972 and, in revised form, September 18, 1972.

AMS (MOS) subject classifications (1970). Primary 53C75; Secondary 50B20, $50 \mathrm{D} 45$.

Key words and phrases. Cyclic order, local cyclic order three, dissection order, general tangent circle, osculating circle.

(c) American Mathematical Society 1973 
This means that if $q, r, s$ are any three points of $A$ and if $C_{q}, C_{r}, C_{s}$ are general osculating circles at those three points then $C_{r}$ separates $C_{q}$ and $C_{s}$ whenever $r$ separates $q$ and $s$ on $A$. Actually this is a simple extension of a known property of arcs of cyclic order three [5]. For a generalization of these ideas see also [1, pp. 22-24], [2, Chapter 4.2] and [3, p. 9].

From Theorem $\mathrm{A}$ it follows at once that a general osculating circle to $A$ at a point $p$ in $A^{\prime}$ separates the endpoints and meets $A$ only at $p$. For any oriented circle $C$ we use the notations $C_{*}$ and $C^{*}$ to denote the regions to the left and right of $C$ respectively. If the general osculating circles are oriented in the usual way, it follows that either (1) $a \in C^{*}$ and $b \in C_{*}$ or (2) $a \in C_{*}$ and $b \in C^{*}$ for all oriented general osculating circles at points of $A^{\prime}$. Arc $A$ is called positive or negative according as condition (1) or condition (2) holds. It may be noted that positive and negative arcs are interchanged by inversion so it is usually sufficient to consider only one of the two cases.

We proceed to develop criteria for the cyclic order $t$ of arc $A$.

\section{Criteria for cyclic order.}

Lemma 1. Let $K$ be any member of the pencil $K$ for arc $A$. Then (1) if $p \in A^{\prime} \cap K$ arc $A$ crosses $K$ at $p$ and $K$ is not a general tangent circle at $p$, and (2) the points of $A$ in $A^{\prime} \cap K$ alternate between the arcs of $K$ determined by $a, b$.

Proof. If $C$ is a general osculating circle at $p$ it was noted above that $C$ separates $a$ and $b$. It follows that no circle tangent to $C$ contains both $a$ and $b$. Thus $K$ cannot belong to the general tangent pencil at $p$ and $A$ must cross $K$. This proves assertion (1). To establish assertion (2) suppose for definiteness that $A$ is a positive arc. Let $K$ be given an arbitrary orientation and let $K_{1}$ and $K_{2}$ be, respectively, the arcs of $K$ from $a$ to $b$ and from $b$ to $a$. If $q \in A^{\prime} \cap K_{1}$ and if $C$ is a general osculating circle at $q$ then by definition of positive arc $a \in C^{*}$ and $b \in C_{*}$. Thus at $q, K_{1}$ crosses from $C^{*}$ to $C_{*}$. This is equivalent to the statement that, at $q, C$, and hence $A$, crosses from $K_{*}$ to $K^{*}$. Thus at all points of $A^{\prime} \cap K_{1}$ arc $A$ crosses $K$ from $K_{*}$ to $K^{*}$. A precisely similar argument shows that at all points of $A^{\prime} \cap K_{2}$ arc $A$ crosses $K$ from $K^{*}$ to $K_{*}$. Since the crossings of $A$ with $K$ must alternate between crossings from $K_{*}$ to $K^{*}$ and crossings from $K^{*}$ to $K_{*}$ it follows that the points of $A^{\prime} \cap K$ must alternate between arcs $K_{1}$ and $K_{2}$. This establishes assertion (2).

Lemma 2. There exists a circle of pencil $\boldsymbol{K}$ meeting $A$ in $t$ points.

Proof. By definition of cyclic order there is a circle $C$ meeting $A$ in $t$ points $q_{1}, \cdots, q_{t}$. If $q_{1}=a, q_{t}=b$, circle $C$ is already the desired circle in $\boldsymbol{K}$. 
If $q_{1} \neq a$, let $M$ be the circle through $a, q_{1}, q_{t}$. Hence $M$ separates the two arcs of $C$ from $q_{1}$ to $q_{t}$. By Lemma 1 applied to the subarc $q_{1} q_{t}$ of $A$ the points $q_{2}, q_{3}, \cdots, q_{t-1}$ alternate between these arcs. Hence each of the $t-3$ subarcs of $A, q_{i} q_{i+1}, i=2, \cdots, t-2$, meets $M$. Thus $M \cap A$ includes these $t-3$ intersections together with $a, q_{1}, q_{t}$, so $M$ is a circle which meets $A$ in $t$ points and contains endpoint $a$. If $q_{t} \neq b$ an exactly similar argument replaces $M$ by a circle $N$ containing both $a$ and $b$ and meeting $A$ in $t$ points. Circle $N$ is then the desired member of $\boldsymbol{K}$.

Lemma 3. Let $R, S$ be members of pencil $\boldsymbol{K}$ meeting $A$ in $r, s$ points respectively. Then $|r-s| \leqq 1$.

Proof. We may assume without loss of generality that $r \geqq s$. Let the points of $A \cap R$ be $a, q_{1}, \cdots, q_{r-2}, b$. By Lemma 1 the points $q_{i}$ alternate between the arcs of $R$ from $a$ to $b$. But since $S$ separates these two arcs, $S$ must meet each of the $r-3$ subarcs of $A, q_{i} q_{i+1}, i=1, \cdots, r-3$. Thus $A \cap S$ contains at least $a, b$, and these $r-3$ intersections. That is, $s \geqq r-1$. Since we have assumed $r \geqq s$ this means that $|r-s|=r-s \leqq 1$.

THEOREM 1. If circle $C$ is a member of pencil $\boldsymbol{K}$ and is a general tangent circle at $a$ or $b$, then $A^{\prime} \cap C$ contains exactly $t-3$ points.

Proof. Let $s$ be the number of points in $A^{\prime} \cap C$, whence the number of points in $A \cap C$ is $s+2$. It is known [6, Theorem 1.1] that if a circle meets an arc of cyclic order $t$ in $t$ points, it cannot be a general tangent circle at any of them. Hence $A \cap C$ cannot contain as many as $t$ points and $s+2<t$. But by Lemma $3, t-(s+2) \leqq 1$. These two inequalities imply $s=t-3$ which was to be proved. An alternate proof of Theorem 1 could be given along the following lines. Let $K$ be a member of $K$ meeting $A$ in $a, q_{1}, \cdots, q_{t-2}, b$ as guaranteed by Lemma 2 . Because of Lemma 1 , if a variable circle starts from $K$ and varies monotonically in $K$ the intersections $q_{i}$ all vary in the same direction on $A$. If the variation is chosen so the $q_{i}$ are displaced toward $a$ it can be continued until $q_{1}$ reaches $a$ in which case the circle is a general tangent circle at $a$ and meets $A^{\prime}$ in $t-3$ points. Similarly if the variation displaces the $q_{i}$ toward $b$ it continues until $q_{t-2}$ reaches $b$, yielding the general tangent circle at $b$. Actually these two tangent circles divide pencil $K$ into two segments such that all circles in one segment meet $A$ in $t$ points while all circles in the other segment meet it in $t-1$ points. This latter segment may consist of a single circle, however.

The following result is a simple but useful corollary.

THEOREM 2. A necessary and sufficient condition that $A$ have cyclic order three is that there exist a circle $K \in \boldsymbol{K}$ meeting $A$ only at $a, b$. 
Proof. If the cyclic order of $A$ is three, the circle $C$ of Theorem 1 satisfies the required condition since $t-3=0$. Conversely, suppose $K$ is a circle meeting $A$ only at endpoints $a, b$. By Lemma 2 there is a circle $L$ through the endpoints meeting $A$ in $t$ points where $t$ is the cyclic order. By Lemma 3 applied to $K$ and $L$, we find $|t-2| \leqq 1$ whence $t \leqq 3$. It follows that $t=3$ since the cyclic order is never less than three.

\section{Dissection order.}

Definition. The dissection order of the arc $A$ is the minimum number of arcs of cyclic order three into which it can be decomposed.

\section{THEOREM 3. If the arc $A$ has dissection order $d$, then $d+2 \leqq t \leqq 3 d$.}

Proof. By hypothesis, $A$ can be decomposed into $d$ arcs, each of cyclic order three. Since no circle can meet any of these $d$ arcs in more than three points, no circle can meet $A$ in more than $3 d$ points. Thus $t \leqq 3 d$, and the right-hand inequality in the theorem holds.

Consider now the circle $C$ of pencil $K$ which belongs to the tangent pencil to $A$ at $a$. By Theorem $1, A^{\prime} \cap C$ contains exactly $t-3$ points, say $q_{1}, q_{2}, \cdots, q_{t-3}$. Thus $C$ cuts $A$ into the $t-2$ subarcs $a q_{1}, q_{1} q_{2}, \cdots, q_{t-3} b$. Since $C$ meets each of these subarcs only at its endpoints, it follows by Theorem 2 that these arcs are all of cyclic order three. Thus $A$ has been divided into $t-2$ arcs, each of cyclic order three. This means that, by definition of dissection order, $d \leqq t-2$. This is equivalent to the left-hand inequality in this theorem.

It is natural to ask whether the result of Theorem 3 is, in some sense, a best possible one. The answer is in the affirmative. If $d$ and $t$ are any positive integers satisfying the inequalities of Theorem 3, there exists an arc $A$ of local cyclic order three having cyclic order $t$ and dissection order $d$. The proof of this, however, requires more machinery than has been developed here.

\section{REFERENCES}

1. J. Haller, Über ordnungsminimale Bogen bzw. Kurven in der Ebene und ihre kParatingenten, Bayer. Akad. Wiss. Math.-Natur. Kl. S.-B. 1963, 15-25. MR 30 \#2411.

2. O. Haupt and H. Kunneth, Geometrische Ordnungen, Die Grundlehren der math. Wissenschaften, Band 133, Springer-Verlag, Berlin and New York, 1967. MR 37 \#3491.

3. —_ Bemerkungen zu Sätzen von Herrn S. B. Jackson über ebene Bogen von der zyklischen Ordnung drei, Bayer. Akad. Wiss. Math.-Natur. Kl. S.-B. 1971, 1-11.

4. N. Lane and P. Scherk, Characteristic and order of differentiable points in the conformal plane, Trans. Amer. Math. Soc. 81 (1956), 358-378. MR 18, 64.

5. N. D. Lane, K. D. Singh and P. Scherk, Monotony of the osculating circles of arcs of cyclic order three, Canad. Math. Bull. 7 (1964), 265-271. MR 28 \#5389. 
6. S. B. Jackson, A note on arcs of finite cyclic order, Proc. Amer. Math. Soc. 12 (1961), 364-368. MR 24 \#A2873.

7. —_, Arcs of local cyclic order three, Math. Scand. 28 (1971), 355-365.

8. - Arcs of local cyclic order three with prescribed end conditions, Technical Report TR 69-89, University of Marlyand, Department of Mathematics, College Park, Md., 1969.

9. A. Marchaud, Sur les continus d'ordre borné, Acta Math. 55 (1930), 67-115.

Department of Mathematics, University of Maryland, College Park, MaryLAND 20742 